\title{
Efeito da hidrotermia em abacate 'Hass' sobre a capacidade antioxidante, compostos fenólicos e coloração
}

\section{Effect of hydrothermally 'Hass" avocado about antioxidant capacity, total phenolic content and coloration}

\author{
Maria Augusta Tremocoldi ${ }^{1}$; Érica Regina Daiuto ${ }^{2 *}$; \\ Severino Matias de Alencar ${ }^{3}$; Rogério Lopes Vieites ${ }^{4}$
}

\section{Resumo}

Este trabalho teve por objetivo determinar a atividade antioxidante, teor de fenólicos totais e coloração em abacate 'Hass' submetido ao tratamento hidrotérmico. Os frutos foram submetidos ao tratamento hidrotérmico a $45^{\circ} \mathrm{C}$ durante $5,10,15$ e 20 minutos. Após o tratamento, os frutos foram armazenados em temperatura ambiente $\left(21^{\circ} \mathrm{C} \pm 1\right.$ e $70 \pm 5 \%$ de umidade relativa) e sob refrigeração $\left(10^{\circ} \mathrm{C} \pm 1\right.$ e $90 \pm 5 \%$ de umidade relativa). Os frutos foram analisados quanto à capacidade antioxidante pelo método DPPH e compostos fenólicos totais aos 0, 3, 9 e 12 dias. A coloração dos frutos foi avaliada aos $0,3,6,9,12$ e 15 dias. Os frutos controle apresentaram maior capacidade antioxidante e teor de compostos fenólicos totais ao longo do período de armazenamento, quando comparados aos frutos submetidos ao tratamento hidrotérmico. A hidrotermia alterou o comportamento quanto à manutenção da atividade antioxidante em relação aos frutos controle. Apesar de valores superiores de atividade antioxidante para os frutos mantidos a $21^{\circ} \mathrm{C} \pm 1$ e $70 \pm 5 \%$ de umidade relativa, aqueles refrigerados apresentaram melhor aspecto para comercialização. Os valores de luminosidade, cor a* e b* diminuíram com os dias de armazenamento. Valores superiores de cor foram observados para os frutos controle e mantidos sob refrigeração.

Palavras-chave: Persea Americana Mill, refrigeração, pós-colheita

\begin{abstract}
This study aimed to determine the antioxidant activity, total phenolic compounds and color in avocado 'Hass' hydrothermally treated. The fruits were hydrothermally treated at $45^{\circ} \mathrm{C}$ for 5, 10, 15 and $20 \mathrm{~min}$. After treatment, fruit were stored at room temperature $\left(21 \pm 1^{\circ} \mathrm{C}\right.$ and $70 \pm 5 \%$ relative humidity) and cold $\left(10^{\circ} \mathrm{C} \pm 1\right.$ and $90 \pm 5 \%$ relative humidity). The fruits were analyzed for their antioxidant capacity by DPPH method and phenolic compounds at $0,3,9$ and 12 days. The fruits color was measured at $0,3,6$, 9,12 and 15 days. The control fruits had higher antioxidant capacity and content of phenolic compounds during the storage period, compared to the fruits hydrothermally treated. The hydrothermally treatment altered the behavior as for the maintenance of the antioxidant activity in relation to the fruits control. In spite of superior values of antioxidant activity for the fruits maintained at $21 \pm 1^{\circ} \mathrm{C}$ and $70 \pm 5 \%$ relative humidity, those refrigerated presented better aspect for commercialization. The refrigerated fruits presented better aspect for commercialization in relation to the maintained under room temperature. The brightness, color $\mathrm{a} *$ and $\mathrm{b} *$ values decreased with the storage days. Values color superiors were observed for the fruits control and those maintained under refrigeration. As it increased the irradiation dose reduced the fruits antioxidant activity and coloration.
\end{abstract}

Key words: Persea Americana Mill, refrigeration, postharvest

\footnotetext{
${ }^{1}$ Discente, M.e pelo curso Horticultura, Faculdade de Ciências Agronômicas, Universidade Estadual Paulista "Julio de Mesquita Filho", UNESP, Botucatu, SP. E-mail: mariaaugustatremocoldi@yahoo.com.br

${ }^{2}$ Pesquisadora, Pós Doutoranda, Faculdade de Ciências Agronômicas, UNESP, Botucatu, SP. E-mail: erdaiuto@uol.com.br

${ }^{3}$ Prof. Livre Docente, Universidade de São Paulo, Escola Superior de Agricultura Luiz de Queiroz, USP, SP. E-mail: alencar@, esalq.usp.br

${ }^{4}$ Prof. Titular na Faculdade de Ciências Agronômicas, UNESP, Botucatu, SP. E-mail: vieits@fca.unesp.br

* Autor para correspondência
} 


\section{Introdução}

O abacate (Persea americana Mill.) é fruto climatérico cujo amadurecimento ocorre poucos dias após a colheita (HARDENBURG; WATADA; WANG, 1986; SEYNOUR; TUCKER, 1993). No mercado atual, têm sido valorizadas as variedades de calibres menores, como a Hass, e a perecibilidade destes frutos representa entrave à comercialização (DAIUTO et al., 2010a).

Os estudos relacionados ao aumento do período de conservação de abacate referem-se à avaliação da temperatura de armazenamento, uso de atmosfera modificada com aplicação de cera, irradiação gama e tratamento térmico para prevenção de sintomas de injúria pelo frio (ZAUBERMAN, 1973; CASTRO; BLEIROTH, 1982; SEYMOUR; TUCKER, 1993; GERMANO; ARTHUR; WIENDL, 1996; OLIVEIRA et al., 2000; SANCHES, 2006; MORGADO, 2007; DONADON, 2009). O efeito da hidrotermia em abacates 'Hass' foi avaliada em pós-colheita como forma de inibição da atividade da enzima polifenoloxidase (DAIUTO;VIEITES, 2008) e sobre a atividade respiratória desta variedade (DAIUTO et al., 2010b), sendo observado redução na atividade da enzima e alteração no padrão respiratório.

O abacate possui considerável qualidade nutritiva, com alto conteúdo de fibras, proteínas, sais minerais, destacando-se o potássio e vitaminas, especialmente a vitamina E (USDA, 2007). As frutos e vegetais contêm compostos com potencial antioxidante, como vitaminas $\mathrm{C}$ e $\mathrm{E}$, carotenóides, clorofilas, e antioxidantes fitoquímicos como compostos fenólicos simples, glicosídeos e flavonóides (PELLEGRINI et al., 2007).

Pesquisas envolvendo compostos antioxidantes oriundos de fontes naturais têm sido desenvolvidas em diferentes centros de estudos devido a sua importância na prevenção do desencadeamento das reações oxidativas, tanto nos alimentos como no organismo animal. Esses podem agir retardando ou prevenindo a oxidação do substrato envolvido nos processos oxidativos impedindo a formação de radicais livres (HALLIWELL et al., 1995). Wang, Terrell e Bostic (2010) mencionam que estudos sobre a composição fitoquímica de abacates são escassos e não há conhecimento sobre o teor de compostos fenólicos totais e capacidade antioxidante em diferentes raças e cultivares de abacate. Além disso, estes parâmetros não foram ainda avaliados ao longo do período de armazenamento dos abacates 'Hass'.

Dessa maneira, objetivou-se na avaliação da atividade antioxidante, teor de compostos fenólicos totais e coloração em abacates 'Hass' submetidos ao tratamento hidrotérmico.

\section{Matérias e Métodos}

Foram utilizados frutos de abacate 'Hass' fornecidos pela empresa Jaguacy, localizada em Bauru/ SP, cujas coordenadas geográficas são: latitude $22^{\circ} 19^{\prime} 18^{\prime \prime} \mathrm{S}$, longitude $49^{\circ} 04^{\prime} 13^{\prime \prime} \mathrm{W}$ e $526 \mathrm{~m}$ de altitude, distante $90 \mathrm{~km}$ de Botucatu: latitude de $22^{\circ} 52^{\prime} 20^{\prime \prime} \mathrm{S}$, longitude $48^{\circ} 26^{\prime} 37^{\prime \prime} \mathrm{W}$ e $815 \mathrm{~m}$ de altitude. Os frutos foram colhidos no ponto de maturação fisiológica, de acordo com o teor de óleo e selecionados visando à homogeneização do lote quanto ao tamanho, cor e ausência de injúrias e defeitos.

Os frutos foram transportados ao Instituto de Pesquisa (IPEN) e submetidos ao tratamento hidrotérmico a $45^{\circ} \mathrm{C}$ durante $5,10,15$ e 20 minutos. Cada tratamento foi constituído de 15 frutos. Frutos não submetidos à hidrotermia caracterizaram o controle do experimento. Após serem submetidos ao tratamento os frutos foram armazenados em temperatura ambiente a $21^{\circ} \mathrm{C} \pm 1$ e $70 \pm 5 \%$ de umidade relativa e câmara frigorífica a $10^{\circ} \mathrm{C} \pm 1$ e $90 \pm 5 \%$ de umidade relativa, no Laboratório de Frutas e Hortaliças da Faculdade de Ciências Agronômicas da UNESP de Botucatu.

Foi utilizado o delineamento inteiramente casualizado, em esquema fatorial ( $5 \times 3$ e $5 \times 5)$, 
com três repetições. O primeiro fator composto pelos tratamentos realizados aos frutos submetidos ao tratamento térmico. O segundo fator consistiu de 3 tempos para atividade antioxidante e teor de compostos fenólicos totais e cinco tempos de avaliação (3, 6, 9, 12 e 15 dias) para a coloração. A parcela experimental foi formada por três abacates.

\section{Análises}

Todas as análises foram realizadas em triplicata.

Preparo do extrato etanólico da polpa para determinação da atividade antioxidante e fenólicos totais

A extração das amostras foi feita de acordo com o método descrito por Kähkönen, Hopia e Vuorela (1999), com algumas adequações. Foi utilizada a mistura de solventes etanol: água $(80: 20 \mathrm{v} / \mathrm{v})$ para a extração. Os extratos dos frutos foram obtidos em triplicata. Pesaram-se 3,0 g da polpa do fruto em tubos tipo falcon onde foram adicionados $30 \mathrm{~mL}$ da mistura etanol: água $(80: 20 \mathrm{v} / \mathrm{v})$. Os tubos contendo a polpa do fruto e o solvente foram submetidos à trituração com a temperatura ambiente $\left(21^{\circ} \mathrm{C}\right)$. Em seguida, os extratos foram centrifugados a 5000xg durante 15 minutos. Na sequência os extratos foram filtrados e armazenados em frascos escuros à temperatura de $8^{\circ} \mathrm{C}$, até o momento das análises, e por período não superior a uma semana.

\section{Compostos fenólicos totais}

O teor de compostos fenólicos totais foi determinado pelo método espectrofotométrico de Folin-Ciocateau utilizando ácido gálico como padrão de referência. Uma alíquota de $0,5 \mathrm{~mL}$ do extrato etanólico foi adicionada a $2,5 \mathrm{~mL}$ do reagente Folin Ciocalteau diluído em água destilada (1:10). Em seguida foram adicionados $2 \mathrm{~mL}$ de carbonato de sódio a 4\%. As leituras foram feitas após $2 \mathrm{~h}$ de incubação no escuro, em espectrofotômetro
(UV Mini 1240, Shimadzu Co.) em $740 \mathrm{~nm}$. Os resultados dos compostos fenólicos totais foram expressos em equivalente de ácido gálico, com base em uma curva de calibração de ácido gálico com concentrações variando de 5 a $100 \mu \mathrm{g} /$ $\mathrm{mL}$. As análises foram realizadas em triplicata (SINGLETON; ORTHOFER; LAMUELA, 1999).

\section{Atividade antioxidante pelo método DPPH}

A atividade antioxidante foi determinada por meio da capacidade sequestrante do radical livre DPPH (2,2 difenil-1-picril-hidrazil) segundo Mensor et al. (2001). Uma alíquota de $500 \mu \mathrm{L}$ do extrato etanólico foi colocada em tubo de ensaio contendo $3 \mathrm{~mL}$ de etanol absoluto e $300 \mu \mathrm{L}$ do radical DPPH em solução de etanol $0,5 \mathrm{mM}$ e incubada por 45 minutos, em temperatura ambiente $\left(21^{\circ} \mathrm{C}\right)$ e ao abrigo da luz. A atividade anti-radical foi determinada na forma de atividade antioxidante $(\mathrm{AA})$, pela equação: AA $(\%)=100-\{[(\mathrm{Aa}-\mathrm{Ab})$ x 100 ] /Ac \}.Onde: Aa = absorbância da amostra; $\mathrm{Ab}=$ absorbância do branco; $\mathrm{Ac}=$ absorbância do controle negativo.

O controle negativo foi feito substituindo-se o volume do extrato por igual volume do solvente utilizado na extração. O branco foi preparado substituindo o volume da solução de DPPH por igual volume de solvente.

\section{Coloração da polpa}

A coloração foi medida em colorímetro da marca Konica Minolta (Chroma meter, CR 400/410) A cor foi expressa pelo sistema de coordenadas retangulares L, a* b* conforme a Comission Internatinale de E'clairage (CIE) onde L expressa em porcentagem valores de luminosidade $(0 \%=$ negro e $100 \%=$ branco), a* representa as cores vermelha $(+)$ ou verde $(-)$ e b* as cores amarela $(+)$ ou azul (-). A leitura foi feita na polpa dos frutos em 3 pontos diferentes. 
Os resultados foram submetidos a análises de variância e as médias foram comparadas pelo teste de Tukey $(\mathrm{P}<0,05)$.

\section{Resultados e Discussões}

O valor mínimo encontrado de teor de compostos fenólicos foi de 33,0 e o valor máximo foi de 60 mg ácido gálico. $100 \mathrm{~g}^{-1}$ polpa (Tabela1). O teor de compostos fenólicos totais da polpa dos frutos de abacate submetidos à hidrotermia, nas duas temperaturas de armazenamento, alterou-se durante o período experimental.

Tabela 1. Compostos fenólicos totais ( $\mathrm{mg}$ ácido gálico. $\left.100 \mathrm{~g}^{-1} \mathrm{polpa}\right) \mathrm{em}$ abacates 'Hass' submetidos ao tratamento hidrotérmico e armazenados sob refrigeração $\left(10^{\circ} \mathrm{C} \pm 1\right.$ e $90 \pm 5 \%$ de UR $)$ e temperatura ambiente $\left(21^{\circ} \mathrm{C} \pm 1\right.$ e $70 \pm 5 \%$ de UR), Botucatu-SP, 2010.

\begin{tabular}{|c|c|c|c|}
\hline \multicolumn{4}{|c|}{ Dia } \\
\hline \multicolumn{4}{|c|}{ Refrigeração } \\
\hline Tratamento & 3 & 9 & 12 \\
\hline $0 \mathrm{mim}$ & $46 \mathrm{~A} \pm 9,7$ & $57 \mathrm{~A} \pm 5,7$ & $40 \mathrm{~A} \pm 5,4$ \\
\hline $5 \mathrm{~min}$ & $34 \mathrm{~A} \pm 0,9$ & $39 \mathrm{~B} \pm 1,9$ & $50 \mathrm{~A} \pm 7,3$ \\
\hline $10 \mathrm{~min}$ & $34 \mathrm{~A} \pm 6,5$ & $40 \mathrm{AB} \pm 2,5$ & $44 \mathrm{~A} \pm 3,2$ \\
\hline $15 \mathrm{~min}$ & $41 \mathrm{~A} \pm 3,3$ & $37 \mathrm{~B} \pm 3,1$ & $40 \mathrm{~A} \pm 2,4$ \\
\hline $20 \mathrm{~min}$ & $33 \mathrm{~A} \pm 5,0$ & $48 \mathrm{AB} \pm 7,8$ & $47 \mathrm{~A} \pm 9,2$ \\
\hline
\end{tabular}

$\mathrm{P}<0,001$.

Letras maiúsculas comparam médias de tratamento para cada dia.

Médias seguidas de pelo menos 1 letra em comum não diferem estatisticamente.

\begin{tabular}{cccc}
\hline & & Ambiente & \\
\hline Tratamento & $\mathbf{3}$ & $\mathbf{9}$ & $\mathbf{1 2}$ \\
\hline 0 mim & $42 \mathrm{a} \pm 6,2$ & $53 \mathrm{a} \pm 5,3$ & $56^{\mathrm{a}} \pm 18,1$ \\
$5 \mathrm{~min}$ & $33 \mathrm{~b} \pm 4,5$ & $54 \mathrm{ab} \pm 2,0$ & $62 \mathrm{a} \pm 13,8$ \\
$10 \mathrm{~min}$ & $35 \mathrm{~b} \pm 1,9$ & $52 \mathrm{ab} \pm 8,4$ & $60 \mathrm{a} \pm 1,9$ \\
$15 \mathrm{~min}$ & $41 \mathrm{a} \pm 8,8$ & $49 \mathrm{a} \pm 3,3$ & $44 \mathrm{a} \pm 7,2$ \\
$20 \mathrm{~min}$ & $40 \mathrm{a} \pm 5,0$ & $60 \mathrm{a} \pm 1,2$ & $52 \mathrm{a} \pm 12,3$ \\
\hline
\end{tabular}

$\mathrm{P}=0,01$

Letras minúsculas comparam médias de dia para cada tratamento

Médias seguidas de pelo menos 1 letra em comum não diferem estatisticamente.

Fonte: Elaboração dos autores.

Para os frutos refrigerados observou-se diferença estatística significativa no dia 9 de armazenamento, sendo que aqueles do tratamento controle apresentaram valores superiores aos demais tratamentos, diferindo estatisticamente dos frutos imersos por 5 minutos e 15 minutos, os quais apresentaram teores menores de compostos fenólicos. Para o terceiro e $12^{\circ}$ dia não houve diferença significativa entre as médias dos frutos nos tratamentos.
Observou-se para maioria dos tratamentos uma tendência de aumento dos teores de compostos fenólicos até os 12 dias de armazenamento. Para os frutos imersos por 5 e 10 minutos, os compostos fenólicos aumentaram durante todo o período de armazenamento. Esta tendência mesma tendência foi observada em frutos de atemóia submetidos ao tratamento térmico e armazenados a $8^{\circ} \mathrm{C}$, onde teores elevados destes compostos foram registrados no $12^{\circ}$ dia (TORRES, 2008). Segundo o autor, 
este fato pode estar relacionado com o tratamento térmico antes do armazenamento.

Para os frutos imersos por 15 minutos, ocorreu decréscimo do terceiro para o nono dia havendo acréscimo no teor dos compostos para o $12^{\circ}$ dia de armazenamento. Torres (2008) observou que os frutos de atemóia armazenados a $15^{\circ} \mathrm{C}$ apresentaram queda dos compostos fenólicos após o $3^{\circ}$ dia, havendo aumento destes compostos apenas no $12^{\circ}$ dia de armazenamento. Os frutos tratados termicamente e armazenados a $15^{\circ} \mathrm{C}$ também apresentaram queda após o $3^{\circ}$ dia e novo aumento no $15^{\circ}$ dia, quando estes já não se encontravam aptos ao consumo.

Nos frutos mantidos em temperatura ambiente não foi observada diferença significativa com relação às médias dos frutos nos tratamentos dentro dos dias de análise. Para os dias de armazenamento em cada tratamento, observou-se diferença estatística significativa do terceiro para o $12^{\circ}$ dia de armazenamento para os frutos imersos por 5 e 10 minutos. Os teores de compostos fenólicos nestes tratamentos mantiveram-se no nono dia, não havendo diferença significativa, aumentando no $12^{\circ}$, diferindo dos demais tratamentos onde não houve diferença significativa durante o período de armazenamento.

A composição dos compostos fenólicos em frutas pode ser modificada pelo ambiente $\mathrm{e}$ fatores pós-colheita, incluindo armazenamento e processamento. $\mathrm{O}$ processamento e armazenamento prolongados promovem oxidação enzimática e química dos compostos fenólicos, contribuindo para a sua redução (KAUR; KAPOOR, 2001). Muitos estudos têm mostrado que os compostos fenólicos geralmente diminuem em frutos climatérios, como tomates, bananas, mangas e goiabas durante o amadurecimento (HAARD; CHISM, 1996; LAKSHMINARAYANA; SUBHADRA; SUBRAMANYAM, 1970; MITRA; BALDWIM, 1997; SELVARAJ; KUMAR, 1989). Já Rodriguez, Lopez e Garcia (2010) em pesquisa com amora, maracujá, goiaba e mamão, relataram que os compostos fenólicos aumentaram durante o amadurecimento dos frutos, concordando com Lima, Mélo e Lima (2002) que trabalhando com pitangas, em dois estádios de amadurecimento do fruto, verificaram que os frutos amadurecidos apresentaram maiores teores de compostos fenólicos quando comparados aos frutos no início do amadurecimento.

Ainda pode-se mencionar que para os frutos mantidos a temperatura ambiente, o aumento no teor de compostos fenólicos durante o armazenamento poderia estar associado à perda de massa das frutas, concentrando estas substâncias (ANTUNES; GONÇALVEZ; TREVISAN, 2006), já que conforme relatado por Daiuto et al. (2010b) os frutos mantidos à temperatura ambiente apresentam maior percentual de perda de massa em relação àqueles refrigerados.

No presente trabalho, nos frutos do tratamento controle nota-se aumento dos teores de compostos fenólicos ao longo do armazenamento até o nono dia nas duas condições de armazenamento. Daiuto et al. (2010b) nas mesmas condições deste estudo, encontraram que o pico respiratório dos frutos de abacate Hass ocorreu a partir do nono dia de armazenamento. Portanto, neste experimento a redução da concentração do teor de compostos fenólicos pode ser devido a este fato, ou seja, devido o pico climatério seguido do início do processo de senescência. Já para o aumento dos teores destes compostos nos frutos mantidos em temperatura ambiente, a perda de massa pode ter sido responsável pela concentração deste compostos, conforme já mencionado.

Analisando a média geral dos tratamentos, apesar de não ser observada diferença significativa entre tratamento, observou-se que os frutos controle obtiveram maiores teores de compostos fenólicos em relação aos demais tratamentos, nas duas condições de armazenamento, indicando que a hidrotermia pode ter alterado o comportamento dos frutos. 
Para atividade antioxidante nas duas condições de armazenamento, não ocorreu diferença entre as médias dos valores encontrados na polpa dos frutos dos diferentes tratamentos em cada dia e nem dos dias em cada tratamento (Tabela 2).

Tabela 2. Atividade antioxidante DPPH• em abacates 'Hass' submetidos ao tratamento hidrotérmico e armazenados sob refrigeração $\left(10^{\circ} \mathrm{C} \pm 1\right.$ e $90 \pm 5 \%$ de UR) e temperatura ambiente $\left(21^{\circ} \mathrm{C} \pm 1\right.$ e $70 \pm 5 \%$ de UR), Botucatu-SP, 2010.

\begin{tabular}{|c|c|c|c|}
\hline \multicolumn{4}{|c|}{ Dia } \\
\hline \multicolumn{4}{|c|}{ Refrigeração } \\
\hline Tratamento & 3 & 9 & 12 \\
\hline $0 \mathrm{mim}$ & $35,3 \pm 9,7$ & $57,1 \pm 33,6$ & $66,0 \pm 19,0$ \\
\hline $5 \mathrm{~min}$ & $37,4 \pm 15,3$ & $52,2 \pm 4,7$ & $58,2 \pm 14,9$ \\
\hline $10 \mathrm{~min}$ & $27,0 \pm 8,4$ & $38,4 \pm 6,4$ & $41,5 \pm 10,7$ \\
\hline $15 \mathrm{~min}$ & $39,1 \pm 13,4$ & $37,3 \pm 5,7$ & $61,4 \pm 14,4$ \\
\hline $20 \mathrm{~min}$ & $37,6 \pm 12,0$ & $54,6 \pm 29,5$ & $47,1 \pm 13,6$ \\
\hline $\mathrm{p}=0,16$ & & & \\
\hline \multicolumn{4}{|c|}{ Ambiente } \\
\hline Tratamento & 3 & 9 & 12 \\
\hline $0 \mathrm{mim}$ & $17,4 \pm 5,8$ & $57,3 \pm 11,2$ & $58,1 \pm 28,2$ \\
\hline $5 \mathrm{~min}$ & $50,9 \pm 16,1$ & $51,0 \pm 10,3$ & $33,6 \pm 15,0$ \\
\hline $10 \mathrm{~min}$ & $51,7 \pm 10,4$ & $56,8 \pm 8,1$ & $39,8 \pm 24,9$ \\
\hline $15 \mathrm{~min}$ & $44,8 \pm 16,6$ & $46,9 \pm 9,5$ & $46,2 \pm 28,5$ \\
\hline $20 \mathrm{~min}$ & $37,9 \pm 19,0$ & $53,5 \pm 4,9$ & $15,3 \pm 4,0$ \\
\hline $\mathrm{p}=0,06$ & & & \\
\hline
\end{tabular}

Fonte: Elaboração dos autores.

Observou-se que a atividade antioxidante para os frutos mantidos sob refrigeração aumentou para todos os tratamentos com o decorrer dos dias de armazenamento, com exceção dos frutos imersos por 20 minutos a $45^{\circ} \mathrm{C}$. Os frutos deste tratamento apresentaram aumento do terceiro para o nono dia de armazenamento, havendo decréscimo para o décimo segundo dia de armazenamento. Rodríguez, Lopez e Garcia (2010) trabalhando com amora, maracujá, goiaba e mamão, observaram que a capacidade antioxidante aumentou durante o amadurecimento dos frutos. Daiuto et al. (2011) observaram que frutos de abacate 'Fuerte' amadurecidos, ou seja, aptos ao consumo, apresentaram maior capacidade antioxidante (DPPH) em relação àqueles não amadurecidos ou em senescência.

Melo et al. (2009) citam que durante o tratamento térmico podem ocorrer vários eventos que justificam a alteração da capacidade antioxidante, que pode aumentar, reduzir ou se manter no alimento. O aumento seria devido ao fato do tratamento térmico propiciar a oxidação parcial do composto bioativo que exibe maior habilidade em doar o átomo de hidrogênio ao radical a partir do grupo hidroxil e/ ou a estrutura aromática do polifenol apresentando portanto maior capacidade em suportar o deslocamento do elétron desemparelhado em volta do anel. Além disso, o tratamento térmico pode favorecer a formação de novos compostos, como os produtos da reação de Maillard (redutonas), que têm ação antioxidante (NICOLI; ANESE; PARPINEL, 1999). Pelo fato da refrigeração ser o método mais eficiente no controle do amadurecimento dos frutos, esta pode ter contribuído para a manutenção da capacidade antioxidante destes durante o período de armazenamento.

No terceiro dia os frutos que apresentaram maior capacidade antioxidante foram os imersos 
por 15 minutos, enquanto que no dia 9 foram os frutos do tratamento testemunha. Para o $12^{\circ}$ dia de armazenamento, os frutos do tratamento controle apresentaram maior capacidade antioxidante em relação aos demais tratamentos. Verificouse que durante os doze dias de armazenamento não ocorreu diferença significativa entre os frutos dos diferentes tratamentos em cada dia e nem dos dias em cada tratamento. Os frutos do tratamento controle testemunha foram os que apresentaram maior capacidade antioxidante ao longo do período de armazenamento, em média 52,8\%. Em estudo realizado por Melo et al. (2008) os extratos de frutas que exibiram percentual entre 50 e $70 \%$ e abaixo de $50 \%$ foram classificados como extratos de moderada e fraca capacidade de sequestro, respectivamente.

A média da atividade antioxidante encontrada foi de 46\%. Melo et al. (2008), ao avaliarem a capacidade antioxidante de frutas pelo método DPPH obtiveram atividade antioxidante inferior a $50 \%$ para manga espada e melancia. Prado (2009) ao avaliar a atividade antioxidante de frutas tropicais, pelo método $\mathrm{DPPH}$, obteve atividade antioxidante de $58 ; 43,5$ e $35,8 \%$, respectivamente para os extratos de maracujá, melão e manga.

Observou-se que a atividade antioxidante para os frutos mantidos à temperatura ambiente aumentou para todos os tratamentos do terceiro para o nono dia de armazenamento. Para os frutos imersos por 5,10 e 20 minutos a $45^{\circ} \mathrm{C}$ houve decréscimo da capacidade antioxidante do nono dia para o $12^{\circ}$, concordando com Moreira (2009) que ao avaliar a capacidade antioxidante de melões Cantaloupe minimamente processado observou diminuição da atividade antioxidante com o decorrer dos dias de armazenamento.

Kaur e Kapoor (2001) afirmam que os compostos antioxidantes de ocorrência natural podem ser significativamente perdidos como consequência de processamento e armazenamento afetando, dessa forma, a capacidade antioxidante do alimento. No caso do tratamento térmico, a redução da ação antioxidante pode ocorrer quando este promove a destruição do composto bioativo e/ou a formação de novos compostos com ação pró-oxidante (NICOLI; ANESE; PARPINEL, 1999).

Para os frutos imersos por 15 minutos (T4), a atividade manteve- se inalterada, havendo acréscimo no tratamento testemunha (T1).

No terceiro dia os frutos que apresentaram maior capacidade antioxidante foram os frutos imersos por 10 minutos, enquanto que no dia 9 foram os frutos controle. Para o $12^{\circ}$ dia de armazenamento, os frutos do tratamento testemunha continuaram apresentando maior capacidade antioxidante em relação aos demais tratamentos. Verificou- se que durante os doze dias de armazenamento não ocorreu diferença significativa entre os frutos dos diferentes tratamentos em cada dia e nem dos dias em cada tratamento. Os frutos imersos por 10 minutos (T3) foram os que apresentaram maior capacidade antioxidante ao longo do período de armazenamento, em média 49,4\%. A média da atividade antioxidante encontrada foi de $47,8 \%$, a mesma encontrada para os frutos mantidos sob refrigeração.

Os resultados de cor da polpa, referentes ao L, dos frutos de abacate tratados por hidrotermia nas duas condições de armazenamento mostraram que ocorreu diferença entre as médias dos frutos nos dias de armazenamento, sem diferença entre os tratamentos (Tabela 3).

Nos frutos refrigerados observou-se em todos os tratamentos que a polpa dos frutos apresentou elevada luminosidade durante todo o período de armazenamento, concordando com Donadon (2009), que avaliou a coloração d a polpa de abacate 'Hass', durante 21 dias e mantidos a $5^{\circ} \mathrm{C}$ e $10^{\circ} \mathrm{C}$, encontrando valores de 82,76 e 80,88 , respectivamente para as duas temperaturas. Já em temperatura ambiente constatou-se diminuição dos valores de luminosidade durante todo o período de armazenamento, concordando com Donadon (2009). As polpas dos frutos mantidos em temperatura ambiente, apresentaram em 
média valores de luminosidade inferiores àqueles refrigerados. Notou-se também valores de luminosidade superiores para o tratamento controle nas duas temperaturas de armazenamento.
Ocorreu diferença entre as médias dos valores de cor a* obtidas na polpa dos frutos nas duas condições de armazenamento durante o período experimental (Tabela 4).

Tabela 3. Luminosidade (\%) em abacates 'Hass' submetidos ao tratamento hidrotérmico e armazenados sob refrigeração $\left(10^{\circ} \mathrm{C} \pm 1\right.$ e $90 \pm 5 \%$ de UR) e temperatura ambiente $\left(21^{\circ} \mathrm{C} \pm 1\right.$ e $70 \pm 5 \%$ de UR), Botucatu-SP, 2010.

\begin{tabular}{|c|c|c|c|c|c|c|c|}
\hline \multicolumn{8}{|c|}{ Dia } \\
\hline \multirow[b]{2}{*}{ Tratamento } & \multicolumn{6}{|c|}{ Refrigeração } & \multirow[b]{2}{*}{ Média } \\
\hline & $\mathbf{0}$ & 3 & 6 & 9 & 12 & 15 & \\
\hline $0 \mathrm{mim}$ & $88,0 \pm 11,3$ & $87,9 \pm 3,3$ & $89,35 \pm 1,5$ & $88,9 \pm 1,4$ & $86,5 \pm 0,8$ & $86,7 \pm 5,0$ & $84,5 \pm 8,1$ \\
\hline $5 \min$ & $88,0 \pm 11,3$ & $83,9 \pm 1,4$ & $85,2 \pm 0,9$ & $81,3 \pm 2,0$ & $85,6 \pm 0,7$ & $83,8 \pm 2,9$ & $81,3 \pm 7,5$ \\
\hline $10 \mathrm{~min}$ & $88,0 \pm 11,3$ & $86,5 \pm 1,9$ & $85,1 \pm 2,0$ & $82,1 \pm 0,9$ & $85,2 \pm 2,8$ & $82,5 \pm 2,7$ & $81,6 \pm 7,7$ \\
\hline $15 \mathrm{~min}$ & $88,0 \pm 11,3$ & $84,1 \pm 0,3$ & $87,1 \pm 1,5$ & $81,5 \pm 2,8$ & $85,7 \pm 1,5$ & $87,8 \pm 2,6$ & $82,4 \pm 8,1$ \\
\hline $20 \mathrm{~min}$ & $88,0 \pm 11,3$ & $86,8 \pm 0,9$ & $84,9 \pm 1,4$ & $83,7 \pm 1,8$ & $83,8 \pm 3,4$ & $84,4 \pm 1,9$ & $81,9 \pm 7,7$ \\
\hline Média & $88,0 \mathrm{~b} \pm 9,6$ & $85,3 a \pm 1,8$ & $85,6 a \pm 1,6$ & $82,1 \mathrm{a} \pm 1,9$ & $85,1 \mathrm{a} \pm 2,2$ & $84,7 a \pm 3,0$ & \\
\hline
\end{tabular}

Houve somente efeito de dia $(\mathrm{p}<0,001)$.

Letras minúsculas comparam médias gerais de dia.

Médias seguidas de pelo menos 1 letra em comum não diferem estatisticamente.

\begin{tabular}{|c|c|c|c|c|c|c|c|}
\hline \multirow[b]{2}{*}{ Tratamento } & \multicolumn{6}{|c|}{ Ambiente } & \multirow[b]{2}{*}{ Média } \\
\hline & $\mathbf{0}$ & 3 & 6 & 9 & 12 & 15 & \\
\hline $0 \mathrm{mim}$ & $88,0 \pm 11,3$ & $85,6 \pm 1,1$ & $86,0 \pm 1,9$ & $85,9 \pm 2,1$ & $89,1 \pm 0,7$ & $76,9 \pm 4,9$ & $81,9 \pm 7,9$ \\
\hline $5 \mathrm{~min}$ & $88,0 \pm 11,3$ & $85,1 \pm 1,9$ & $84,0 \pm 1,5$ & $83,3 \pm 1,7$ & $78,9 \pm 2,5$ & $80,3 \pm 6,1$ & $79,9 \pm 7,5$ \\
\hline $10 \mathrm{~min}$ & $88,0 \pm 11,3$ & $87,4 \pm 1,2$ & $85,8 \pm 1,4$ & $82,8 \pm 3,8$ & $79,6 \pm 8,2$ & $72,1 \pm 17,6$ & $79,3 \pm 10,7$ \\
\hline $15 \mathrm{~min}$ & $88,0 \pm 11,3$ & $85,6 \pm 3,0$ & $83,7 \pm 0,8$ & $81,4 \pm 1,5$ & $79,4 \pm 3,0$ & $75,6 \pm 6,4$ & $79,0 \pm 7,6$ \\
\hline $20 \mathrm{~min}$ & $68,0 \pm 11,3$ & $85,0 \pm 1,5$ & $82,3 \pm 1,4$ & $74,5 \pm 4,3$ & $83,1 \pm 4,4$ & $77,4 \pm 0,8$ & $78,4 \pm 7,5$ \\
\hline Média & $88,0 \mathrm{c} \pm 9,6$ & $85,8 \mathrm{a} \pm 2,0$ & $83,9 a b \pm 1,7$ & $80,5 \mathrm{ab} \pm 4,5$ & $80,3 a b \pm 4,6$ & $76,4 b c \pm 9,0$ & \\
\hline
\end{tabular}

Houve somente efeito de dia $(\mathrm{p}<0,001)$.

Letras minúsculas comparam médias gerais de dia.

Médias seguidas de pelo menos 1 letra em comum não diferem estatisticamente.

Fonte: Elaboração dos autores.

O parâmetro de cor $a^{*}$ negativo indica a presença do componente de cor verde dos frutos. Do mesmo modo que para luminosidade, os valores de $a^{*}$ mantiveram-se maiores para os frutos armazenados sob temperatura refrigerada. Para todos os tratamentos observa-se tendência de diminuição da intensidade da cor verde com os dias de armazenamento.
Para o parâmetro de cor b* obtido na polpa dos frutos, nas duas condições de armazenamento, observou-se ocorreu diferença entre as médias dos frutos dos diferentes tratamentos nos dias de armazenamento (Tabela 5). 
Tabela 4. Cor a* em abacates 'Hass' submetidos ao tratamento térmico e armazenados sob refrigeração $\left(10^{\circ} \mathrm{C} \pm 1 \mathrm{e}\right.$ $90 \pm 5 \%$ de UR) e temperatura ambiente $\left(21^{\circ} \mathrm{C} \pm 1\right.$ e $70 \pm 5 \%$ de UR), Botucatu-SP, 2010.

\begin{tabular}{ccccccc}
\hline \multicolumn{7}{c}{ Dia } \\
\hline
\end{tabular}

Houve somente efeito de dia $(\mathrm{p}<0,001)$.

Letras minúsculas comparam médias gerais de dia.

Médias seguidas de pelo menos 1 letra em comum não diferem estatisticamente.

\begin{tabular}{cccccccc}
\hline & \multicolumn{7}{c}{ Ambiente } \\
\cline { 2 - 7 } Tratamento & $\mathbf{0}$ & \multicolumn{1}{c}{$\mathbf{3}$} & \multicolumn{1}{c}{$\mathbf{6}$} & $\mathbf{9}$ & $\mathbf{1 2}$ & $\mathbf{1 5}$ & Média \\
\hline 0 mim & $-20,7 \pm 3,7$ & $-12,7 \pm 1,7$ & $-10,9 \pm 1,6$ & $-10,3 \pm$ & $-6,7 \pm 1,3$ & $5,5 \pm 0,8$ & $11,1 \pm 5,4$ \\
\hline 5 min & $-20,7 \pm 3,7$ & $-10,0 \pm 2,7$ & $-8,2 \pm 4,2$ & $-7,3 \pm 1,4$ & $-5,4 \pm 2,8$ & $-2,5 \pm 1,6$ & $-9,0 \pm 6,4$ \\
10 min & $-20,7 \pm 3,7$ & $-8,0 \pm 1,7$ & $-5,3 \pm 0,4$ & $-9,8 \pm 1,1$ & $-4,5 \pm 4,3$ & $-5,3 \pm 1,4$ & $-8,9 \pm 6,1$ \\
15 min & $-20,7 \pm 3,7$ & $-9,2 \pm 1,6$ & $-6,3 \pm 1,5$ & $-9,8 \pm 1,3$ & $-3,2 \pm 3,7$ & $-3,8 \pm 3,5$ & $-8,8 \pm 6,5$ \\
20 min & $-20,7 \pm 3,7$ & $-10,3 \pm 0,9$ & $-7,4 \pm 1,8$ & $-2,9 \pm 2,6$ & $-4,8 \pm 1,4$ & $-3,9 \pm 0,4$ & $-8,3 \pm 6,5$ \\
\hline Média & $-20,7 \mathrm{~d} \pm 3,2$ & $-9,4 \mathrm{c} \pm 1,8$ & $-6,8 \mathrm{abc} \pm 2,4$ & $-7,5 \mathrm{bc} \pm 3,3$ & $-4,5 \mathrm{ab} \pm 2,9$ & $-3,9 \mathrm{a} \pm 2,0$ & \\
\hline
\end{tabular}

Houve somente efeito de dia $(\mathrm{p}<0,001)$.

Letras minúsculas comparam médias gerais de dia.

Médias seguidas de pelo menos 1 letra em comum não diferem estatisticamente.

Fonte: Elaboração dos autores.

Tabela 5. Cor $\mathrm{b}^{*}$ em abacates 'Hass' submetidos ao tratamento térmico e armazenados sob refrigeração $\left(10^{\circ} \mathrm{C} \pm 1 \mathrm{e}\right.$ $90 \pm 5 \%$ de UR) e temperatura ambiente $\left(21^{\circ} \mathrm{C} \pm 1\right.$ e $70 \pm 5 \%$ de UR $)$, Botucatu-SP, 2010.

\begin{tabular}{cccccccc}
\hline \multicolumn{7}{c}{ Defrigeração } \\
\hline Tratamento & \multicolumn{1}{c}{$\mathbf{0}$} & \multicolumn{1}{c}{$\mathbf{3}$} & $\mathbf{6}$ & $\mathbf{9}$ & $\mathbf{1 2}$ & $\mathbf{1 5}$ & Média \\
\hline $\mathbf{0}$ mim & $43,5 \pm 3,7$ & $42,7 \pm 4,5$ & $35,3 \pm 1,1$ & $39,0 \pm 3,1$ & $44,0 \pm 2,7$ & $35,0 \pm 3,5$ & $39,9 \pm 4,1$ \\
\hline 5 min & $43,5 \pm 3,7$ & $42,0 \pm 1,9$ & $39,0 \pm 5,0$ & $38,5 \pm 4,5$ & $42,2 \pm 0,7$ & $39,2 \pm 2,5$ & $40,8 \pm 3,5$ \\
10 min & $43,5 \pm 3,7$ & $41,4 \pm 1,7$ & $41,6 \pm 4,6$ & $41,2 \pm 2,0$ & $38,8 \pm 1,1$ & $35,4 \pm 2,4$ & $40,3 \pm 3,6$ \\
15 min & $43,5 \pm 3,7$ & $43,3 \pm 1,0$ & $42,0 \pm 1,1$ & $37,4 \pm 2,2$ & $39,8 \pm 0,6$ & $34,7 \pm 6,3$ & $40,1 \pm 4,3$ \\
20 min & $43,5 \pm 3,7$ & $40,7 \pm 1,9$ & $39,9 \pm 3,1$ & $41,3 \pm 4,1$ & $40,0 \pm 3,5$ & $36,7 \pm 3,5$ & $40,4 \pm 3,5$ \\
Média & $43,5 \mathrm{a} \pm 3,2$ & $41,8 \mathrm{ab} \pm 1,7$ & $40,6 \mathrm{abc} \pm 3,5$ & $39,6 \mathrm{bc} \pm 3,4$ & $40,2 \mathrm{abc} \pm 2,1$ & $36,5 \mathrm{c} \pm 3,9$ & \\
\hline
\end{tabular}

Houve somente efeito de dia $(\mathrm{p}<0,001)$.

Letras minúsculas comparam médias gerais de dia.

Médias seguidas de pelo menos 1 letra em comum não diferem estatisticamente.

\begin{tabular}{|c|c|c|c|c|c|c|c|}
\hline \multicolumn{8}{|c|}{ Ambiente } \\
\hline Tratamento & $\mathbf{0}$ & 3 & 6 & 9 & 12 & 15 & Média \\
\hline $0 \mathrm{mim}$ & $43,5 \mathrm{aA} \pm 3,7$ & $45,9 \mathrm{aA} \pm 4,3$ & $39,4 a A \pm 0,6$ & $39,4 a A \pm 3,9$ & $36,7 \mathrm{aA} \pm 1,0$ & $40,4 \mathrm{aA} \pm 5,0$ & $40,9 \pm 3,3$ \\
\hline $5 \mathrm{~min}$ & $43,5 \mathrm{aA} \pm 3,7$ & $39,8 \mathrm{aA} \pm 3,0$ & $38,9 \mathrm{aA} \pm 3,4$ & $37,6 \mathrm{aA} \pm 3,7$ & $37,0 \mathrm{aA} \pm 2,1$ & $36,4 \mathrm{aAB} \pm 3,2$ & $38,9 \pm 3,7$ \\
\hline $10 \mathrm{~min}$ & $43,5 \mathrm{aA} \pm 3,7$ & $38,2 \mathrm{aA} \pm 0,9$ & $42,1 \mathrm{aA} \pm 2,1$ & $40,2 \mathrm{aA} \pm 2,1$ & $38,1 \mathrm{aA} \pm 3,7$ & $27,8 \mathrm{bB} \pm 10,8$ & $38,3 \pm 6,8$ \\
\hline $15 \mathrm{~min}$ & $43,5 \mathrm{aA} \pm 3,7$ & $40,3 \mathrm{aA} \pm 1,3$ & $43,4 \mathrm{aA} \pm 3,8$ & $39,1 \mathrm{aA} \pm 1,5$ & $37,2 \mathrm{aA} \pm 3,7$ & $40,5 \mathrm{aA} \pm 5,6$ & $40,7 \pm 3,8$ \\
\hline $20 \mathrm{~min}$ & $43,5 \mathrm{aA} \pm 3,7$ & $39,2 \mathrm{abA} \pm 2,4$ & $45,3 \mathrm{aA} \pm 2,2$ & $34,2 \mathrm{bA} \pm 1,4$ & $39,5 \mathrm{abA} \pm 2,3$ & $43,2 \mathrm{abA} \pm 5,4$ & $40,8 \pm 4,6$ \\
\hline Média & $43,5 \pm 3,2$ & $39,4 \pm 2,0$ & $42,4 \pm 3,5$ & $37,8 \pm 3,1$ & $38,0 \pm 2,8$ & $37,0 \pm 8,4$ & \\
\hline
\end{tabular}

Houve efeito da interação tempo x dia $(\mathrm{p}=0,02)$.

Letras minúsculas comparam médias de dia em cada tempo.

Letras maiúsculas comparam médias de tempo em cada dia.

Médias seguidas de pelo menos 1 letra em comum não diferem estatisticamente.

Fonte: Elaboração dos autores. 
O parâmetro de cor $b^{*}$ positivo indica a presença do componente de cor amarelo nos frutos. Os valores de cor $b^{*}$ mostraram-se superiores para os frutos armazenados sob refrigeração. Ocorreu diminuição da intensidade deste componente com os dias de armazenamento para os frutos mantidos sob refrigeração ou temperatura ambiente.

A Tabela 6 apresenta as correlações entre os parâmetros avaliados. Existem correlações fracas e moderadas entre as variáveis.

Tabela 6. Correlação linear para os parâmetros avaliados de atividade antioxidante, fenólicos totais e cor (BotucatuSP, 2010).

\begin{tabular}{llllll}
\hline & \multicolumn{1}{c}{$\begin{array}{c}\text { Atividade } \\
\text { antioxidante }\end{array}$} & Fenólicos totais & Luminosidade & Cor a* & Cor b* \\
\hline $\begin{array}{l}\text { Atividade } \\
\text { antioxidante }\end{array}$ & 1,000 & & & & \\
Fenólicos totais & 0,2582 & 1,0000 & & & \\
Luminosidade & 0,21918 & -02447 & 1,0000 & & \\
Cor a* & 0,1646 & 0,3827 & 0,2305 & 1,0000 & \\
Cor $\mathbf{b}^{*}$ & $-0,3423$ & $-0,4391$ & 0,1739 & $-0,5441$ & 1,0000 \\
\hline
\end{tabular}

Fonte: Elaboração dos autores.

Para os parâmetros de cor observou-se pequena correlação. À medida que diminuíram os valores de $b^{*}$ (verde) ressaltou-se a tonalidade da cor $a^{*}$ (amarela), justificando o amarelecimento da polpa.

Para atividade antioxidante e compostos fenólicos totais a correlação foi baixa. Podedo-se inferir que a atividade antioxidante na polpa dos frutos, determinados por está metodologia (DPPH), não foi devida aos compostos fenólicos. Já Daiuto et al. (2011) avaliando a atividade antioxidante também por DPPH e compostos fenólicos em abacates 'Fuerte' mostram que não houve correlação significativa entre estes parâmetros. O conteúdo de componentes fenólicos totais não necessariamente estar envolvido na quantificação da atividade antioxidante, assim como mencionado por JacoboVelasquéz; Cisneros-Zevallos 2009). Já ArancibiaAvila et al. (2008) encontraram uma correlação de 0,98 entre o conteúdo de compostos fenólicos e a capacidade antioxidante. Os autores concluíram que o alto teor de polifenóis foi principal responsável pela capacidade antioxidante. Wang, Terrell e Bostic (2010) encontram correlação significativa para o conteúdo de fenólicos totais e a AA $(>=a$ 0,79). Estes dois parâmetros avaliados pelos autores não correlacionaram com o conteúdo de clorofila e carotenóides $(r<0,1)$. Para os autores a alta correlação entre as procianidinas, o conteúdo de polifenóis e a atividade antioxidante sugere que a procianidina é o polifenol principal que contribui para a capacidade antioxidante em abacate.

Daiuto et al. (2011) ressaltam que a presença e outros compostos presentes no fruto, como a vitamina E, devem ser avaliados, a fim de verificar sua contribuição para a atividade antioxidante. Outros métodos de determinação de atividade antioxidante também devem ser avaliados em abacate Hass.

Observou-se correlação moderada também entre os compostos fenólicos, luminosidade e cor $\mathrm{b}^{*}$. Salveit (1997) cita que o escurecimento da polpa de frutos relacionado à degradação de compostos fenólicos pela enzima polifenoloxidase.

\section{Conclusões}

Os frutos controle do tratamento testemunha e mantidos sob refrigeração apresentaram maior capacidade antioxidante e teor de compostos 
fenólicos totais ao longo do período de armazenamento.

A hidrotermia alterou a fisiologia dos frutos alterando o comportamento quanto à manutenção da atividade antioxidante em relação aos frutos controle.

Os valores de luminosidade, cor $\mathrm{a}^{*} \mathrm{e} \mathrm{b}^{*}$ diminuíram com os dias de armazenamento. Valores superiores de cor foram observados para os frutos controle e mantidos sob refrigeração.

\section{Agradecimentos}

À empresa Jaguacy (Bauru-SP) pelo apoio e participação nas pesquisas, e à Coordenação de Aperfeiçoamento de Pessoal de Nível Superior (CAPES), projeto PNPD.

\section{Referências}

ANTUNES, L. E. C.; GONÇALVEZ, E. D.; TREVISAN, R. Alterações de compostos fenólicos e pectina em póscolheita de frutos de amora-preta. Revista Brasileira de Agrociência, Pelotas, v. 12, n. 1, p. 57-61, 2006.

ARANCIBIA-AVILA, P.; TOLEDO, F.; PARK, Y. S.; JUNG, S. T.; KANG, S. G.; HEIO, B. G.; LEE, S. H.; SAJEWICZ, M.; KOWALSKA, T.; GORINSTEIN, S. Antioxidant properties of durian fruit as influenced by ripening. Food Science and Technology, London, v. 41, n. 10, p. 2118-2125, 2008.

CASTRO, J. V.; BLEINROTH, E. W. Conservação do abacate em atmosfera controlada e à temperatura ambiente. Boletim do Instituto de Tecnologia de Alimentos, Campinas, v. 19, n. 2, p. 165-182, 1982.

DAIUTO, E. R.; FUMES, J.; VIEITES, R. L.; CÁBIA, N. C.; CASTRO, R. S. D. Antioxidant capacity and total phenolic content of hidrothermally-treatd 'Fuerte' Avocado. Advances in Horticultural Science, Firenze, v. 25, n. 2, p. 75-80, 2011.

DAIUTO, E. R.; VIEITES, R. L.; TREMOCOLDI, M. A.; VILEIGAS, D. F. Estabilidade físico-química de um produto de abacate acondicionado em diferentes embalagens e conservado pelo frio. Alimentos e Nutrição, Araraquara, v. 21, n. 1, p. 97-105, 2010a.

DAIUTO, E. R.; VIEITES, R. L. Atividade da peroxidase e polifenoloxidase em abacate da variedade
Hass, submetidos ao tratamento térmico. Revista Iberoamericana de Tecnologia Postcosecha, México, v. 9, n. 2, p. 106-112, 2008.

DAIUTO, E. R.; VIEITES, R. L.; TREMOCOLDI, M. A.; RUSSO, V. C. Taxa respiratória de abacate "Hass" submetido a diferentes tratamentos físicos. Revista Iberoamericana de Tecnologia Postcosecha, México, v. 10, n. 2, p. 101-109, 2010b .

DONADON, J. R. Distúrbio fisiológico provocado pelo frio e prevenção com tratamentos térmicos em abacates. 2009. Tese (Doutorado em Produção Vegetal) - Faculdade de Ciências Agrárias e Veterinárias. Universidade Estadual Paulista Júlio de Mesquita Filho, Jaboticabal.

GERMANO, R. M. A.; ARTHUR, V.; WIENDL, F. M. Conservação pós-colheita de abacates Persea americana Mill., variedades fortuna e quintal, por irradiação. Scientia Agricola, Piracicaba, v. 53, n. 2-3, p. 249-253, 1996.

HAARD, N. F.; CHISM, G. W. Characteristics of edible plant tissues. In: DAMODARAN, S. Fennema's food chemistry. New York: Marcel Dekker, 1996. p. 943-1011.

HALLIWEL, B.; AESCHBACH, R.; LÖLIGER, J.; ARUOMA, O. I. The characterization of antioxidants. Food and Chemical Toxicology, Oxford, v. 33, n. 7, p. 601-17, 1995.

HARDENBURG, R. E.; WATADA. A. E.; WANG, C. Y. The comercial storage of fruits, vegetables, and florist and nursery stocks. Beltsville: USDA, 1986. $130 \mathrm{p}$.

JACOBO-VELÁZQUEZ, D. A.; CISNEROSZEVALLOS, L. Correlations of antioxidant activity versus phenolic content revisited: A new approach in data analysis for food and medicinal plants. Journal of Food Science, New York, v. 74, n. 9, p. R107- R113, 2009.

KÄHKÖNEN, M. P.; HOPIA, A. I.; VUORELA, H. J.; RAUHA, J. P.; KALEVI, P.; KUJALA, T. S.; HEINONEN, M. Antioxidant activity of plant extracts containing phenolic compounds. Journal of Agricultural and Food Chemistry, Easton, v. 47, n. 10, p. 3954-3962, 1999

KAUR, C.; KAPOOR, H. C. Anti- oxidant activity and total phenolic- the millenniun's health. International Journal of Food Science and Technology, Oxford, v. 36, n. 7, p. 703-725, 2001.

LAKSHMINARAYANA, S.; SUBHADRA, N. V.; SUBRAMANYAM, H. Some aspects of developmental physiology of mango fruit. The Journal of Horticultural Science, Ashford, v. 45, n. 1, p. 133-142, 1970.

LIMA, V. L. A. G. de; MÉLO, E. de A.; LIMA, D. E. da S. Fenólicos e carotenóides totais em pitanga. Scientia Agricola, Piracicaba, v. 59, n. 3, p. 447-450, 2002. 
MELO, E. A.; MACIEL, M. I. S.; LIMA, V. L. A. G.; SANTANA, A. P. M. Antioxidant capacity of vegetables submitted to thermal treatment. Nutrire - Revista da Sociedade Brasileira de Alimentação e Nutrição, São Paulo v. 34, n. 1, p. 85-95, 2009.

MELO, E. A.; MELO, E. A.; MACIEL, M. I. S.; LIMA, V. A. G. L.; NASCIMENTO, R. J. Capacidade antioxidante de frutas. Revista Brasileira de Ciências Farmacêuticas, São Paulo, v. 44, n. 2, p. 193-201, 2008.

MENSOR, L. L.; MENEZES, F. S.; LEITÃO, G. G.; REIS, A. S.; DOS SANTOS, T. C.; COUBE, C. S.; LEITÃO, S. G. Screening of brazilian plant extracts for antioxidant activity by the use of DPPH free radical method. Phytotherapy Research, London, v. 15, n. 2, p. 127-130, 2001.

MITRA, S. K.; BALDWIN, E. A. Mango. In: MITRA, S. K. (Ed.). Postharvest physiology storage of tropical and subtropical fruit. New York: CAB Internacional, 1997. p. 85-122.

MOREIRA, G. C. Radiação gama ou antimicrobianos naturais na conservação de melão minimamente processado. 2009. Tese (Doutorado em Horticultura) - Faculdade de Ciências Agronômicas. Universidade Estadual Paulista Júlio de Mesquita Filho, Botucatu.

MORGADO, C. M. A. Conservação pós-colheita de abacates 'Geada' e 'Quintal', em diferentes pontos de colheita, com o uso de diferentes temperaturas $e$ proteções. 2007. Monografia (Trabalho de Conclusão de Curso) - Faculdade de Ciências Agrárias e Veterinárias. Universidade Estadual Paulista, Jaboticabal.

NICOLI, M. C.; ANESE, M.; PARPINEL, M. Influence of processing on the antioxidant properties of fruit and vegetables. Trends Food of Science Technoogy, v. 10, n. 3, p. 94-100, 1999.

OLIVEIRA, M. A. de; SANTOS, C. H.; HENRIQUE, C. M.; DOMINGOS, J. R. D. Ceras para conservação pós colheita de frutos de abacateiro fuerte, armazenados em temperatura ambiente. Scientia Agricola, Piracicaba, v. 57, n. 4, p. 777-780, out./dez. 2000.

PELLEGRINI, N.; COLOMBI, B.; SALVATORE, S.; BRENNA, O. V.; GALAVERNA, G.; DEL RIO, D.; BIANCHI, M.; BENNETT, R. N.; BRIGHENTI, F. Evaluation of antioxidant capacity of some fruit and vegetable foods: efficiency of extraction of a sequence of solvents. Journal of the Science of Food and Agriculture, London, v. 87, n. 1, p. 103-111, 2007.

PRADO,A. Composiçãofenólica e atividade antioxidante de frutas tropicais. 2009. Dissertação (Mestrado em Ciência e Tecnologia de Alimentos) - Escola Superior de Agricultura Luiz de Queiroz. Universidade de São Paulo, Piracicaba.
RODRIGUEZ, L.; LOPEZ, L.; GARCIA, M. Determinación de la composición química y actividad antioxidante en distintos estados de madurez de frutas de consumo habitual en Colombia, mora (Rubus glaucus B.), maracuyá (Passiflora edulis S.), guayaba (Psidium guajava L.) y papayuela (Carica cundinamarcensis J.). ACTA, Bogotá, v. 1, n. 21, p. 16-34, 2010.

SALVEIT, M. E. Physical and physiological changes in minimally processed fruits and vegetables. In: TOMÁSBARBERÁN, F. A.; ROBIN, R. D. (Ed.). Phytochemestry of fruits and vegetables. Oxford: Oxford University Press, RU, 1997. p. 205-220.

SANCHES, J. Efeito de injuries mecânicas na qualidade pós-colheita de abacates. 2006. Tese (Doutorado em Produção Vegetal) - Faculdade de Ciências Agrárias e Veterinárias. Universidade Estadual Paulista, Jaboticabal.

SELVARAJ, Y.; KUMAR, R. Studies on fruit softening enzymes and polyphenol oxidase activity in ripening mango (Mangifera indica L.) fruit. Journal of Food Science and Technology, Mysore, v. 26, n. 4, p. 218-222, 1989.

SEYMOUR, G. B.; TUCKER, G. A. Avocado. In: SEYMOUR, G. B.; TAYLOR, J. E.; TUCKER, G. A. Biochemistry of fruit ripening. London: Chapman \& Hall, 1993. p. 53-76.

SINGLETON, V. L.; ORTHOFER, R.; LAMUELA, R. M. Analysis of total phenols and other oxidation substrates and antioxidants by means of Folin-Ciocalteau reagent. Methods of Enzymology, Orlando, v. 299, n. 1, p. 152-178, 1999.

TORRES, L. M. A. R. Conservação pós-colheita de atemóia cv. "Thompson". 2008. Dissertação (Mestrado em Alimentos e Nutrição) - Faculdade de Ciências Farmacêuticas. Universidade Estadual Paulista Júlio de Mesquita Filho, Araraquara.

USDA, U. S. Department of Agriculture, Agricultural Research Service, USDA National Nutrient Database for Standard Reference, Release 20. Nutrient Data Laboratory, 2007. Available at: <http://www.ars.usda. gov/ba/bhnrc/ndl>. Accessed at: 15 out. 2011.

WANG, W.; TERRELL, R.; BOSTIC, L. G. Antioxidant capacities, procyanidins and pigments in avocados of different strains and cultivars. Food Chemistry, London, v. 122, n. 4, p. 1193-1198, 2010.

ZAUBERMAN, M. S. Susceptibility to chilling injury of three avocado cultivars stages of ripening. HortScience, Alexandria, v. 8, n. 4, p. 511-513, 1973. 